\section{$\underset{\substack{\text { hommes } \\ \text { \& migrations }}}{ }$}

\section{Hommes \& migrations}

Revue française de référence sur les dynamiques

migratoires

$1313 \mid 2016$

1983, le tournant médiatique

\title{
Une mémoire transfrontalière
}

Les escartons du Briançonnais

Anne-Marie Granet-Abisset

\section{OpenEdition \\ 12 Journals}

\section{Édition électronique}

URL : http://journals.openedition.org/hommesmigrations/3578

DOI : 10.4000/hommesmigrations.3578

ISSN : 2262-3353

Éditeur

Musée national de l'histoire de l'immigration

\section{Édition imprimée}

Date de publication : 1 janvier 2016

Pagination : 126-130

ISBN : 978-2-919040-34-6

ISSN : $1142-852 X$

Référence électronique

Anne-Marie Granet-Abisset, « Une mémoire transfrontalière », Hommes \& migrations [En ligne], 1313 |

2016, mis en ligne le 17 juin 2016, consulté le 15 septembre 2020. URL : http://

journals.openedition.org/hommesmigrations/3578 


\section{SPÉCIAL FRONTIÈRES}

\section{UNE MÉMOIRE TRANSFRONTALIÈRE LES ESCARTONS DU BRIANÇONNAIS}

Par ANNE MARIE GRANET-ABISSET, université de Grenoble-Alpes (UMR 5190 LARHRA).

L ongtemps connus des seuls habitants du Briançonnais qui en gardaient la mémoire ou des quelques spécialistes et érudits, les escartons du Briançonnais sont redevenus une référence pour évoquer un territoire transnational. Si cette organisation originale, vivante durant quelques siècles (entre 1343 et 1789), matérialise un exemple achevé d'organisation politique et sociale à cheval sur des territoires appartenant désormais à des nations différentes, il reste que ce système n'est pas unique au sein de l'arc alpin. Dans les territoires d'altitude aux confins des États et des royaumes mais au cœur des relations entre ces derniers, il semble être un modèle courant. N'ayant plus depuis deux siècles d'existence légale, leur prégnance mémorielle fonde en revanche leur existence patrimoniale, voire même légitime, par des rappels à l'histoire, des recompositions transfrontalières intéressantes à analyser.

\section{Des territoires expérimentaux dans le fonctionnement}

En 1343, en concomitance avec le début de l'inexorable construction des États, enrichies par le commerce entre France et Italie, les communautés du Briançonnais, du Queyras, du Valcluson, d'Oulx et de Château-Dauphin, soit au total 51 villages du Briançonnais et du Piémont, rachètent au dauphin Humbert II l'ensemble des droits seigneuriaux, moyennant la somme substantielle de 12000 florins-or et le versement d'une rente annuelle par chaque escarton secondaire'. Cet acte est matérialisé par la signature d'une charte, sous le sceau du dauphin². Ce dernier, ayant vendu sa province au Roi de France, a obtenu que ce titre prestigieux marque l'association de sa province, le Dauphiné, à la couronne. Majeur, cet acte l'est pour l'avenir des communautés durant le temps de son fonctionnement. Celles-ci s'affranchissent, en effet, des redevances financières et de l'essentiel des impôts, notamment l'impôt sur les communaux et sur le cheptel, la ressource essentielle des sociétés de montagne. Les seules redevances restent la gabelle sur le sel (pour l'élevage) et la taille. En même temps, elles obtiennent des franchises municipales importantes et surtout le droit d'administrer leurs affaires communes, cas exemplaire de démocratie locale avant l'heure.

L'ensemble des chefs de famille (y compris les femmes lorsqu'elles sont veuves) élisent chaque année des consuls ( 2 par commune) et nomment des officiers qui agissent sous l'autorité des consuls. Leurs missions principales consistent dans l'organisation des corvées (notamment l'entretien des canaux d'irrigation et des sentiers 
et la prévention des risques naturels et du danger majeur des incendies) indispensables pour le bon fonctionnement de la vie collective et la nécessaire gestion de ces territoires "fragiles". Ils ont aussi à gérer la répartition des impositions, en argent ou en nature, dont la levée des troupes et la distribution des soldats séjournant sur le territoire au sein des familles. Les consuls doivent faire l'avance des impôts à régler au roi. Pour cette raison, cette fonction théoriquement élective et alternante revient régulièrement aux familles les plus aisées. Toutes les communautés sont rassemblées dans le Grand Escarton, dont le siège est à Briançon, chargé de s'occuper des affaires au niveau de l'ensemble territorial, et plus particulièrement de défendre les intérêts communs auprès du Parlement de Grenoble et auprès du roi. C'est là le signe d'une réelle autonomie de gestion qui fonctionnera jusqu'à la Révolution française.

Au nom de l'abolition des privilèges lors de la nuit du 4 août 1789, ces droits particuliers, qui étaient réaffirmés à chaque changement de règne, sont supprimés. Cette décision entérine l'entrée de ces territoires dans la norme générale, et pour ces communautés, un ressentiment devant la perte de leur spécificité et de leur autonomie. Déjà un siècle auparavant, en 1713, lors d'un conflit géopolitique qui ne les concerne que de loin et du traité d'Utrecht qui redéfinit la frontière avec le Duché de Savoie, le Grand Escarton avait subi une première rupture. Les trois escartons piémontais avaient été cédés, fractionnant cet ensemble transfrontalier. Pour autant, la tradition d'autonomie reste forte et les structures de l'escarton perdurent en s'insérant dans les nouvelles, issues de l'organisation de la République et de l'Empire. Les familles qui investissaient la charge de consul assurent dans la continuité les fonctions municipales. L'organisation des corvées, dont certaines se perpétuent jusqu'aux années récentes, reste le signe du maintien actif de cette organisation et de la vitalité de la vie collective, malgré sa suppression théorique.

\section{La mémoire d'un territoire transnational : un usage réactualisé}

Siège de la République des Escartons durant cinq siècles et symbole de cette tradition des Républiques alpines qualifiées par les observateurs extérieurs dans leur lecture dépréciative de "petites Républiques ", voire de "démocraties de la misère ", le Briançonnais incarne particulièrement cette occultation et cette résurgence de la mémoire historique. Si celle-ci peut s'affirmer au sein de la population comme être reprise par les élus, c'est que ces institutions ont laissé dans la mémoire commune la conscience d'avoir été le terreau expérimental de la démocratie locale.

Le rappel à la tradition d'autoadministration est régulièrement revendiqué jusqu'à présent, dès lors que les populations ont l'impression que le pouvoir leur échappe ou qu'on leur impose des décisions. Il est vrai qu'au regard de la construction économique et politique des États, les territoires alpins n'ont pas cessé d'être considérés comme des zones périphériques, enfermées dans les cercles nationaux qui ont contribué à les marginaliser. Cette marginalisation est d'abord géographique puisque ces territoires sont situés aux abords des frontières politiques qui, durant le XXe siècle, ne cessent d'être renforcées tout en faisant l'objet de conflits autour de leur tracé. Elle est aussi économique, puisque l'évolution de l'aménagement des territoires promeut les villes et les vallées rendues plus accessibles par les routes et les chemins de fer nouvellement construits.

La notion de retard, miroir inversé de la modernité, est associée étroitement à ces territoires décrits comme enclavés en dépit de la construction de routes carrossables, mais dont la configuration inscrit la lenteur et les difficultés d'accès par comparaison avec les autres territoires qui participent du monde de la vitesse, innova- 


\section{SPÉCIAL FRONTIÈRES}

tion jamais démentie depuis le XIXe siècle. L'articulation est rapidement faite avec le maintien de formes d'archaïsme qui finissent par désigner ces territoires, dans une lecture qui reste essentiellement celle des touristes et des voyageurs. Ces montagnes « retrouvées " par ces élites urbaines européennes avec le développement du tourisme, domestiquées à leurs usages, sont ainsi enfermées dans des cercles nationaux, contribuant à les marginaliser et surtout à classer leurs habitants du côté du retard et de l'archaïsme.

À l'inverse dans les années récentes, alors que le tourisme est devenue une activité économique de première importance, les Alpes revendiquent leur place non plus seulement comme " terrain de jeu " pour l'Europe, selon l'expression consacrée de Leslie Stephen, ou comme « espace naturel protégé " pour des citadins en mal d'air pur, d'activité physique et de lieux de ressourcement patrimonial, mais comme modèle de régionalisation de l'espace européen, comme expérimentation de politiques transfrontalières pour des régions qui partagent la même " personnalité ", et pas seulement des problèmes similaires. Dans le cadre de la nouvelle organisation économique et politique européenne, cette proximité peut être utilisée pour légitimer leur carte à jouer. La position de "gigantesque commutateur qui agit sur les connexions et les circuits " selon l'expression de Claude Raffestin', donne aux territoires alpins une place de choix entre l'Europe du Nord et l'Europe méditerranéenne. Dire cela, c'est rappeler leur fonction essentielle comme lieu de passage au sein du continent, point de convergence, en des siècles différents, entre les deux parties dynamiques de l'Europe ${ }^{4}$ et pouvant désormais devenir centre de décision et d'impulsion : une manière de revenir sur l'idée classique qui décrit les Alpes comme une barrière infranchissable au nom des conditions géographiques difficiles. De fait, cette lecture de la montagnebarrière a toujours été portée par les populations extérieures, qu'elles pratiquent les Alpes comme terrain d'excursion et de villégiature ou qu'elles en restent éloignées. La configuration des États installe les Alpes dans une position transfrontalière favorable à de possibles recompositions territoriales liées à l'atténuation des frontières dans une Europe en construction, qui affirme par ailleurs très peu sa dimension alpine : une manière de se réinscrire au centre, de quitter les périphéries nationales pour une transnationalité centrale, mais aussi une posture en avance sur l'évolution générale des territoires, comme une avance sur l'histoire, loin des clichés qui les ont longtemps stigmatisés.

La modernité de cette position s'affirme face aux frilosités ou aux replis nationaux. Loin d'être partagée par l'ensemble des habitants, elle est avant tout le fait de certains acteurs, politiques et économiques. Ces derniers voient le moyen de redonner aux Alpes une place qu'ils estiment méritée, inscrivant leur démarche dans une dynamique novatrices. Ces acteurs légitiment leur posture par l'appel à l'histoire, parfois la plus ancienne. Ce recours à un passé commun, parfois durablement interrompu, est clairement revendiqué comme fondement d'un futur à bâtir.

\section{Inspiration et usages politiques d'une histoire régionale}

On n'est plus seulement dans la revendication d'une mémoire permettant d'assumer le passé mais bien dans celle qui est conçue pour 
négocier le futur. Depuis les années 1990, l'Espace Mont-blanc entre Haute-Savoie, Valais et Val d'Aoste, ou les Escartons entre Briançonnais et Piémont portent ces revendications. Moins anecdotiques ou théoriques qu'il n'y paraît, ces manières de concevoir de nouveaux territoires régionaux utilisent les cadres institutionnels de l'Europe. Au sein d'une multitude de structures qui ne cesse de s'enrichir et de se diversifier, il faut citer des programmes de recherche et d'action qui ont favorisé ces mouvements et continuent à le faire, outils particulièrement incitatifs de coopération transnationale en raison des budgets extrêmement importants qui sont proposés et distribués; les programmes Interreg ${ }^{6}$ font partie des plus connus d'entre eux. Les territoires alpins ont su profiter de ces textes pour obtenir des subventions nécessaires à des projets de développement, comme le rappelle en son temps Michel Barnier, un des initiateurs les plus enthousiastes et clairvoyants de cette politique. Dans l'introduction d'une plaquette sur les politiques de I'Union européenne en faveur de la montagne, il affirme : "Grâce au volontarisme de leurs élus et de leurs habitants, beaucoup de zones de montagnes innovent en matière de développement durable et de coopération interrégionale. Elles seront bien placées pour tirer parti des diverses aides européennes prévues pour la période 2000-2006."

Plus intéressant, ces programmes se fondent également sur des pratiques vivantes. C'est le cas bien connu du travail frontalier dont les meilleurs exemples sont la zone genevoise ${ }^{7}$ ou celle du Tessin, créant une réalité transfrontalière, la seule reconnue jusqu'alors. Parfois création ex nihilo jouant de la proximité géographique et culturelle, ces nouveaux cadres veulent redonner une force à des traditions ou à des structures juridiquement éteintes.
Les Escartons du Briançonnais incarnent particulièrement cette éviction et cette résurgence utilisant l'histoire. Occulté pendant des décennies ,le sujet des " petites Républiques " préoccupait seulement un public d'érudits et d'historiens. Leur mise en retrait allait de pair avec l'affirmation de la modernité étatique et touristique. Ce sont d'abord les associations patrimoniales qui se sont emparées du sujet en créant Le Grand Escarton, à l'occasion de la manifestation organisée autour du retour de la charte à Briançon en 1985. Cette association culturelle très vivace mène une politique de visibilité via les pages locales du Dauphiné libéré, puis de publications spécialisées. Ce rappel à l'histoire gagne en parallèle les ouvrages et guides touristiques paraissant sur le territoire, focalisant sur quelques éléments forts : la charte, la démocratie, l'autonomie, l'originalité du système et désormais le partage d'un territoire et d'une culture. En filigrane ressort l'idée d'une période de forte activité et d'intense dynamisme économique, ce qu'ont montré les études historiques. Les élus et les responsables politiques se sont emparés du sujet. Alors qu'ils ne voyaient dans les Escartons que l'image d'un passé pointant le retard de leurs territoires, ils ont trouvé là un moyen d'affirmer leur spécificité et leur place au sein de la région PACA tout en légitimant les réorganisations territoriales à venir. Le sentiment qu'un rapprochement avec le Piémont devient avantageux est une donnée inédite qui s'est affirmée avec les Jeux olympiques de Turin en 2006, organisés en Italie pour l'essentiel mais aussi partiellement dans le Briançonnais. Le dynamisme de la région Piémont est un argument très ferme face à l'éloignement ressenti de la métropole marseillaise. Le projet du Grand Escarton comme cadre de fonctionnement et d'application de différents programmes en sort d'autant plus renforcé. Cette reconstruction ne s'embarrasse pas 


\section{SPÉCIAL FRONTIÈRES}

toutefois d'arrangements étonnants. Ainsi, profitant de programmes patrimoniaux communs (programme Sentinelle des Alpes, sur les fortifications alpines puis programme Vauban) la Haute-Maurienne savoyarde tend à se rattacher à cet ensemble, modifiant la signification historique. Cependant, cette réécriture de l'histoire est surtout intéressante par les discours tenus pour justifier ce rattachement ${ }^{8}$.

Le renversement de position n'est pas seulement lié à la mode ou au goût récents pour l'histoire et le patrimoine. Cet exemple des Escartons, particulièrement éclairant d'un bricolage du temps entre passé et présent, semble correspondre à une nouvelle utilisation de l'histoire au service d'un discours sur le dynamisme de ces hautes vallées qui a comme projet de retrouver la position avantageuse, gommant les siècles de périphérie négative, associée au rappel d'une certaine autonomie. Les objectifs affichés sont clairement ceux du développement touristique, où le patrimoine et l'histoire servent avant tout de caution. Un bel exemple d'une lecture politique du passé et d'un usage économique du patrimoine, le tout au service d'une reconnaissance et de la revendication d'une place transnationale au sein de l'arc alpin.

\section{REVUE \\ hommes \\ DE L'IMMIGRATION \\ \& migrations}

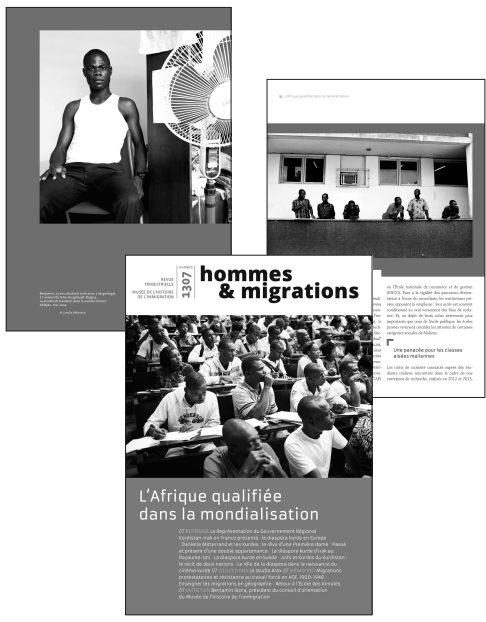

www.hommes-et-migrations.fr

DISPONIBLE EN LIBRAIRIE

ET AU MUSÉE NATIONAL DE L'HISTOIRE DE L'IMMIGRATION 